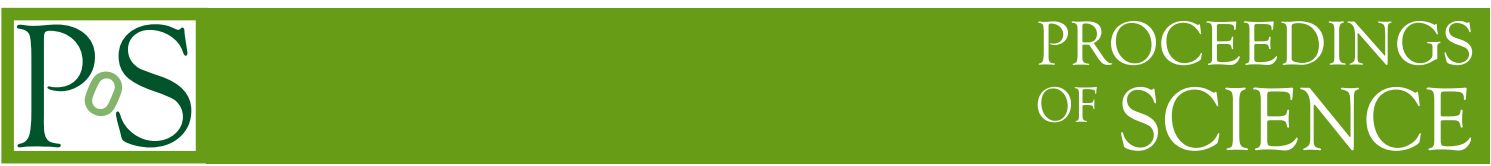

\title{
Searching for a pulsar black hole binary
}

\author{
Ralph Eatough \\ Max-Planck-Institut für Radioastronomie, Auf dem Hügel 69, 53121, Bonn, Germany. \\ E-mail: reatough@mpifr-bonn.mpg.de
}

\begin{abstract}
For tests of General Relativity (GR) and alternative theories of gravity nature has provided perfect "laboratories" in the form of relativistic binary pulsars: pulsars (highly magnetized rapidly rotating neutron stars) orbiting in compact binary systems with either a second neutron star or a white dwarf. In such systems the effects predicted by GR, and some alternative theories of gravity, are significant because of the extreme gravitational fields present. Precise radio timing measurements of the binary pulsar, PSR B1913+16 provided the first evidence for the existence of gravitational waves (GWs) [1]. Since then, the highly relativistic Double Pulsar system, with an orbital period of just 2.4 hours, has provided the most precise test of GR in the so-called "strong field" regime, agreeing with the theory to a level of 0.05 per cent [2]. However, the Double Pulsar is by no means the most extreme binary pulsar system we may expect to find e.g. [3].
\end{abstract}

25th Texas Symposium on Relativistic Astrophysics

December 6-10, 2010

Heidelberg, Germany 


\section{The pulsar black hole binary}

A pulsar orbiting a black hole (PSR-BH) could provide the most stringent tests of GR. The large orbital velocities and component masses in such systems will make the post-Keplerian parameters highly significant (Figure 1 left). The system should also allow measurements of frame dragging caused by the spin of the black hole and, under certain exceptional configurations, it will be possible to measure the quadrupole moment allowing a direct test of the "no hair theorem" in GR (Figure 1 right) [4]. We expect that PSR-BH systems will follow similar evolutionary paths to the current known double neutron star systems (DNS) (viz. small supernova kicks and low systemic velocities) and thus will be found in the Galactic plane.

\section{The detection problem}

Pulsars in relativistic binary systems show changes in their pulse frequency throughout the observation because of the Doppler effect. Standard Fourier based periodicity searches are not sensitive to varying frequency signals. If the observation time is $\sim 1 / 10^{\text {th }}$ of the orbital period the changing pulse frequency can be approximated by a linear drift with time and corrected for by so called "acceleration searches" [5]. Figure 2 shows the improvement in sensitivity using the acceleration method on PSR A in the Double Pulsar system. Since the acceleration of any undiscovered binary pulsar is a priori unknown this must be done for all possible orbital accelerations. Typically, acceleration searches increase the number of computational operations in a pulsar search by at least two orders of magnitude and thus require large-scale computational facilities to perform the data processing.

\section{Current searches}

To find undiscovered relativistic binary pulsars we will analyse the data from the new "High
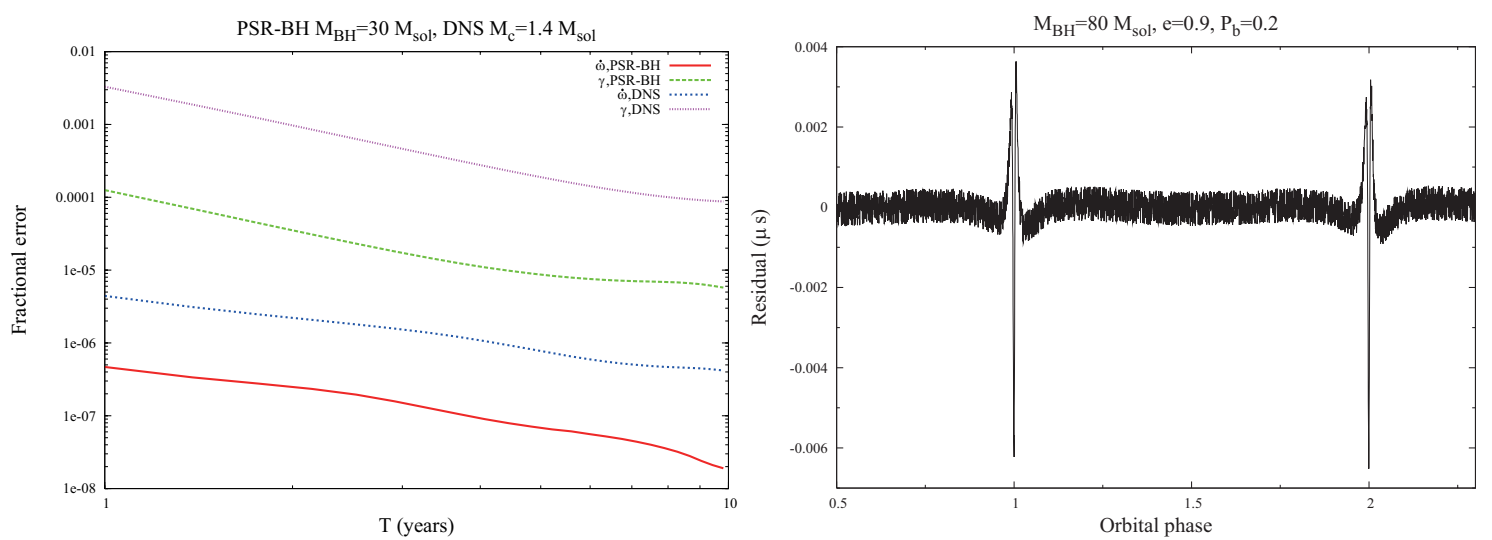

Figure 1: (left) Radio timing precision of two post-Keplerian orbital parameters, and, for simulated PSR$\mathrm{BH}$ and DNS systems. For the same observing scheme, the PSR-BH can yield a better determination of the relativistic motion, ultimately providing an improved test of GR. (right) The simulated signature induced in the post-fit timing residuals by the quadrupole moment of an extreme Kerr BH. Figures provided by Kuo Liu. 

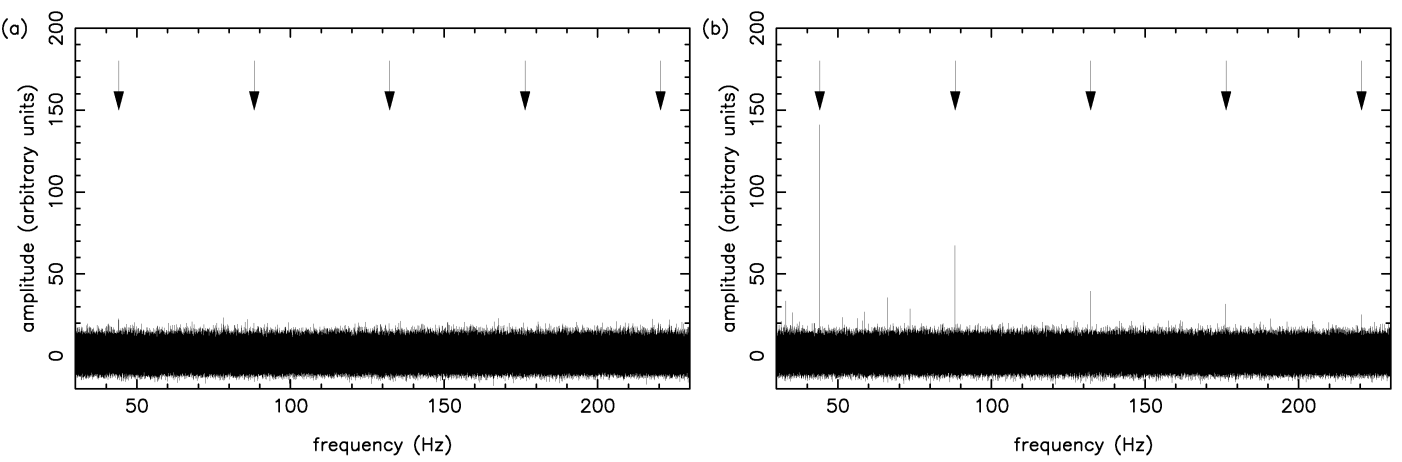

Figure 2: Frequency amplitude spectra of a 17.5 minute observation of the PSR J0737-3039A, in the Double Pulsar system. Arrows mark the fundamental spin frequency and four subsequent harmonics of the pulsar. Panel (a) on the left shows the spectrum without any correction for orbital acceleration while panel (b) on the right shows the spectrum after a correction of $182 \mathrm{~m} \mathrm{~s}^{-2}$ is applied. The acceleration correction makes the detection of the pulsar highly significant.

Time Resolution Universe Pulsar Survey" (HTRU) currently being performed at radio telescopes in Parkes ${ }^{1}$, Australia [6] and at Effelsberg ${ }^{2}$, Germany. The current surveys exploit new technology in field programmable gate arrays to produce digital filterbanks with higher sensitivity to distant millisecond pulsars (see poster contribution by Cherry Ng and Ewan Barr).

For highly relativistic systems, where the orbital period matches the observation time, the acceleration search technique might not be sufficient to correct the binary motion of the pulsar. For these systems, searches in not just orbital acceleration, but three Keplerian orbital parameters must be performed. We will re-analyse the Parkes multi-beam pulsar survey [7], for such extreme systems, in collaboration with the Max Planck Institute for Gravitational Physics (Albert Einstein Institute) using an improved binary search algorithm as described in [8]. Data processing for this search will be performed by the Einstein@ @ome project ${ }^{3}$.

\section{Finding a needle in a haystack!}

The final stage of a pulsar search involves the selection of credible pulsar candidates for followup observations. This task is well suited to the human eye since it involves a process of pattern recognition, however, taking the Effelsberg part of the HTRU survey as an example, we might expect to produce half a billion pulsar candidates; far too many to be examined by eye. It is expected that there will only be a handful of PSR-BH systems visible in the Galaxy e.g. [3]. As such we have been developing artificial neural networks to automatically identify pulsar candidates [9].

\footnotetext{
${ }^{1}$ http: //www.parkes.atnf.csiro.au/

${ }^{2}$ http://www.mpifr-bonn.mpg.de/english/radiotelescope/index.html

${ }^{3}$ http: //einstein.phys.uwm.edu/
} 


\section{Summary}

- Binary pulsars are being used to test GR and alternative theories of gravity, in the strong field, to the highest precision ever.

- A pulsar black hole binary system would allow unprecedented tests of gravitation and enable the properties of the black hole to be investigated.

- Finding such systems requires binary search algorithms that greatly increase the amount of computational power needed for data processing.

- Data from the High Time Resolution Universe Pulsar Survey will be analysed with orbital acceleration search algorithms. Archival data from the Parkes multi-beam pulsar survey will be analysed with sensitive searches for highly relativistic binary pulsars by the Einstein@home project.

- To classify the pulsar candidates generated from the searches, artificial neural networks, trained with large numbers of real and simulated pulsars, will be used.

\section{References}

[1] J. H. Taylor \& J. M. Weisberg, A new test of general relativity - Gravitational radiation and the binary pulsar PSR 1913+16, 1982, ApJ., 253, 908T

[2] M. Kramer, I. H. Stairs, R. N. Manchester, M. A. McLaughlin, A. G. Lyne, R. D. Ferdman, M. Burgay, D. R. Lorimer, A. Possenti, N. D'Amico, J. M. Sarkissian, G. B. Hobbs, J. E. Reynolds, P. C. C. Freire, F. Camilo, Tests of General Relativity from Timing the Double Pulsar, 2006, Science, 314, $97 \mathrm{~K}$

[3] Belczynski, K., Kalogera V., Bulik T., A Comprehensive Study of Binary Compact Objects as Gravitational Wave Sources: Evolutionary Channels, Rates, and Physical Properties, 2002, ApJ, 572, 407B

[4] N. Wex \& S. M. Kopeikin, Frame Dragging and Other Precessional Effects in Black Hole Pulsar Binaries, 1999, ApJ, 514, 388W

[5] S. M. Ransom, J. M. Cordes, S. S. Eikenberry, A New Search Technique for Short Orbital Period Binary Pulsars, 2003, ApJ, 589, 911R

[6] M. J. Keith, A. Jameson, W. van Straten, M. Bailes, S. Johnston, M. Kramer, A. Possenti, S. D. Bates, N. D. R. Bhat, M. Burgay, S. Burke-Spolaor, N. D'Amico, L. Levin, Peter L. McMahon, S. Milia, B. W. Stappers, The High Time Resolution Universe Pulsar Survey - I. System configuration and initial discoveries, 2010, MNRAS, 409, 619K

[7] R.N. Manchester, A.G. Lyne, F. Camilo, J.F. Bell, V.M. Kaspi, N. D’Amico, N.P.F. McKay, F. Crawford, I.H. Stairs, A. Possenti, M. Kramer, D.C. Sheppard, The Parkes multi-beam pulsar survey I. Observing and data analysis systems, discovery and timing of 100 pulsars, 2001, MNRAS, 328, $17 \mathrm{M}$

[8] B. Knispel, B. Allen, J. M. Cordes, J. S. Deneva, D. Anderson, C. Aulbert, N. D. R. Bhat, O. Bock, S. Bogdanov, A. Brazier, F. Camilo, D. J. Champion, S. Chatterjee, F. Crawford, P. B. Demorest, H. Fehrmann, P. C. C. Freire, M. E. Gonzalez, D. Hammer, J. W. T. Hessels, F. A. Jenet, L. Kasian, V. M. 
Kaspi, M. Kramer, P. Lazarus, J. van Leeuwen, D. R. Lorimer, A. G. Lyne, B. Machenschalk, M. A. McLaughlin, C. Messenger, D. J. Nice, M. A. Papa, H. J. Pletsch, R. Prix, S. M. Ransom, X. Siemens, I. H. Stairs, B. W. Stappers, K. Stovall, and A. Venkataraman, Pulsar Discovery by Global Volunteer Computing, 2010, Science, 329, 1305K

[9] R. P. Eatough, N. Molkenthin, M. Kramer, A. Noutsos, M. J. Keith, B. W. Stappers, A. G. Lyne, Selection of radio pulsar candidates using artificial neural networks, 2010, MNRAS, 407, 2443E 